\title{
Practice Patterns for Postoperative Radiation Therapy in Patients with Metastases to the Long Bones: Survey of the Japanese Radiation Oncology Study Group
}

Hikaru Kubota ( $\sim$ hk.0113hu@gmail.com )

Kobe University Hospital

Naoki Nakamura

St. Marianna University School of Medicine

Naoto Shikama

Juntendo University Hospital

Ayako Tonari

Kyorin University Hospital

Hitoshi Wada

Southern TOHOKU Proton Therapy Center

Hideyuki Harada

Shizuoka Cancer Center Hospital

Hisayasu Nagakura

KKR Sapporo medical center

Joichi Heianna

Ryukyu University Hospital

Kei Ito

Tokyo Metropolitan Cancer and Infectious Disease Center Komagome Hospital

Miwako Nozaki

Dokkyo Medical University Saitama Medical Center

Masao Tago

Teikyo University Mizonokuchi Hospital

Masato Fushiki

Nagahama City Hospital

Nobue Uchida

Tokyo Saiseikai Central Hospital

Norio Araki

Kyoto Medical Center

Shuhei Sekii

Kobe University Hospital 


\section{Takashi Kosugi}

Fujieda Municipal General Hospital

\section{Takeo Takahashi}

Saitama Medical University Saitama Medical Center

\section{Terufumi Kawamoto}

Juntendo University Hospital

\section{Tetsuo Saito}

Arao Municipal Hospital

\section{Kazunari Yamada}

Seirei Mikatahara General Hospital

\section{Research}

Keywords: Bone metastases, Long bones, Post-operative radiation therapy, Patterns of practice, Oligometastasis

Posted Date: July 22nd, 2020

DOI: https://doi.org/10.21203/rs.3.rs-45644/v1

License: (c) (i) This work is licensed under a Creative Commons Attribution 4.0 International License. Read Full License

Version of Record: A version of this preprint was published at Journal of Radiation Research on January 18th, 2021. See the published version at https://doi.org/10.1093/jrr/rraa133. 


\section{Abstract}

\section{Background}

Because of the lack of evidence on practice patterns of postoperative radiation therapy (PORT)in patients with metastases to the long bones, it is essential to characterize the current practice patterns and identify factors affecting dose-fractionation to aid future clinical trials.

\section{Methods}

The palliative radiation therapy subgroup of the Japanese Radiation Oncology Study Group conducted an internet-based survey to determine the prescription practices and various dose-fractionations used for PORT. Additionally, responders were asked to recommend dose-fractionations in four hypothetical cancer cases, wherein each case represented a patient with impending pathological fractures and one of the four features: limited prognosis, solitary metastasis, radio-resistant primary tumor, or long-term survival. Responders were asked to indicate their preferred irradiation fields and the reasons for choosing longcourse radiotherapy over short-course radiotherapy (RT).

Results

Eighty-nine radiation oncologists from 67 institutions and 151 RT plans were included. Twenty-two different dose-fractionations were used; the most commonly used and recommended dose-fractionation was $30 \mathrm{~Gy}$ in 10 fractions. "Local control" was most the common reason for preferring longer course RT. While fractionated higher-dose regimens were preferred in case of oligometastasis, low dose regimens were preferred in case of limited prognosis; single fraction RT was never preferred. Most respondents recommended involvement of "the entire orthopedic prosthesis."

\section{Conclusion}

For PORT of metastases to the long bones, $30 \mathrm{~Gy}$ in 10 fractions for the entire orthopedic prosthesis is preferred in current practice. Oligometastasis and a limited prognosis influence the selection of high- and low-dose regimens, respectively. Single fraction RT is never preferred.

\section{Background}

Bone metastases (BMs) are a frequent complication of cancer [1-3], and cause variable symptoms such as pain, pathologic fractures, and spinal cord compression. These symptoms negatively affect the patient's quality of life (QOL) and diminish performance status (PS). Radiation therapy (RT) is recognized as a highly effective standard therapy for BMs providing pain relief in approximately $60-80 \%$ patients as per the evidence from randomized trials [4], with slightly lower response rates (55\%) as per the real-world evidence [5]. 
BMs of the long bones can result in pathologic fractures. Surgical interventions are often required to stabilize the long bones with impending or existing pathological fractures, which may provide pain relief as well as restore functional status [6]. In general, one of the following surgical strategies is commonly used: endoprosthetic reconstruction or internal fixation with either intramedullary nailing or plate/screw fixation devices. Indication of postoperative RT (PORT) after endoprosthetic reconstruction is equivocal because it has been reported to cause prevention of new bone formation in case of proximal femur metastasis [7]. Meanwhile, PORT for metastases to the long bones is commonly practiced, and a few retrospective studies have reported that it lessens pain, improves functional status, decreases reoperation, and slows down further local progression of metastases [8-11]. Further, these studies proposed PORT as one of the effective treatment options. However, the optimal dose-fractionation as well as irradiation fields of PORT have not been reported.

Further, due to the lack of clear evidence, clinical trials of PORT for indication of metastases to the long bones would be essential in the future. While conducting a clinical trial, it is important to understand the radiation oncologists' decision-making patterns and the existing standards of care. To our knowledge, no previous studies have focused on these practice patterns in the PORT for indication of metastases to the long bones.

Thus, the purpose of this study was to characterize the current practice patterns in the PORT for indication of metastases to the long bones and to identify factors that affect the dose-fractionation.

\section{Methods}

Palliative radiation therapy subgroup of the Japanese Radiation Oncology Study Group (JROSG) conducted an internet-based survey among the members of JROSG who are practicing radiation oncologists. The respondents were asked to provide their name, institution, and years of experience in radiation oncology practice as well as the frequency of performing PORT in each institution for the indication of metastases to the long bones in 2017 along with the actual administered dosefractionation. They were also asked for the dose-fractionation they would recommend in each of the four hypothetical cancer cases provided in the study.

The hypothetical cases described impending pathological fractures in the lower limb due to BM, but differed in factors such as nature of malignancy, number of metastases and survival (Table 1). While Case 1 (relatively limited survival) described a case with impending pathological fractures in the lower limb from non-small cell lung cancer with multiple metastases, Case 2 (presence of oligometastasis) investigated whether management for a case of solitary BM would be different from that of Case 1. Case 3 (presence of radio-resistant primary tumor) was identical with Case 2 except for the radio-resistant primary tumor: renal cell carcinoma (RCC). Case 4 (expectation of long survival) involved a patient with BM from breast cancer who was expected to survive longer. Additionally, radiation oncologists who did not recommend short-course RT (e.g., 8 Gy in 1 fraction or 20 Gy in 5 fractions) for Case 1 were asked to explain why they considered long-course RT to be superior to short-course RT. 
Table 1

Hypothetical cases

\section{Case Patients with relatively limited survival}

1

65-year-old man with squamous cell lung cancer had been treated by radical surgery 1 year earlier. Patient had right femoral pain, and examination shows lytic BM in right femoral bone, multiple lung mets and right adrenal met. Internal fixation was performed for right femoral BMs due to the impending fracture. He now has a little pain. His ECOG PS is 1.

Case Patient with oligometastasis

2

65-year-old man with squamous cell lung cancer had been treated by radical surgery 1 year earlier. Patient had right femoral pain, and examination shows solitary lytic BM in right femoral bone. Internal fixation was performed for right femoral BM due to the impending fracture. He now has a little pain. His ECOG PS is 1.

Case Patient with the radio-resistant primary tumor

3

65-year-old man with renal cell carcinoma had been treated by radical surgery 1 year earlier. Patient had right femoral pain, and examination shows lytic BM in right femoral bone and multiple lung. Internal fixation was performed for right femoral BM due to the impending fracture. He now has a little pain. His ECOG PS is 1.

Case4 Patient with expected long survival

50-year-old woman had right femoral pain. Examination showed left breast tumor and multiple lytic lesions including right femoral bone. She was diagnosed as breast cancer (ER positive/PR positive/Her-2 negative/Ki-67 5\%) and multiple BMs. Internal fixation was performed for right femoral BM due to the impending fracture. She now has a little pain. Her ECOG PS is 2.

For analysis, the actual prescribed dose-fractionation as well as the recommended dose-fractionation for the four hypothetical cases were classified into following five categories: "single fraction", "fractionated low dose", "30 Gy in $10 \mathrm{fr}$ ", "fractionated intermediate dose", and "fractionated high dose" as per the biologically effective dose (BED10) (Table 2). "Single fraction" included 14.4 Gy (BED10), "fractionated low dose" included $\geq 22.5$ Gy and $\leq 37.5$ Gy (BED10), "30 Gy in 10 fr" included 39 Gy (BED10), "fractionated intermediate dose" included $\geq 39.2$ Gy and $\leq 59.5$ Gy (BED10), and "fractionated high dose" included $\geq 60$ Gy (BED10). 
Table 2

Classification of dose-fractionations prescribed dose-fractionation used in each institution and recommended for hypothetical cases

\begin{tabular}{|c|c|c|c|}
\hline Total dose (Gy) & No. of fractions & Dose per fraction (Gy) & BED10 \\
\hline \multicolumn{4}{|l|}{ Single fraction } \\
\hline 8 & 1 & 8 & 14.4 \\
\hline \multicolumn{4}{|c|}{ Fractionated low dose } \\
\hline 15 & 3 & 5 & 22.5 \\
\hline 20 & 5 & 4 & 28 \\
\hline 20 & 4 & 5 & 30 \\
\hline 24 & 6 & 4 & 33.6 \\
\hline 25 & 5 & 5 & 37.5 \\
\hline \multicolumn{4}{|l|}{30 Gy in 10 fractions } \\
\hline 30 & 10 & 3 & 39 \\
\hline \multicolumn{4}{|c|}{ Fractionated intermediate dose } \\
\hline 28 & 7 & 4 & 39.2 \\
\hline 24 & 3 & 8 & 43.2 \\
\hline 36 & 12 & 3 & 46.8 \\
\hline 37.5 & 15 & 2.5 & 46.88 \\
\hline 30 & 5 & 6 & 48 \\
\hline 40 & 16 & 2.5 & 50 \\
\hline 39 & 13 & 3 & 50.7 \\
\hline 42 & 14 & 3 & 54.6 \\
\hline 40 & 10 & 4 & 56 \\
\hline 45 & 18 & 2.5 & 56.25 \\
\hline 45 & 15 & 3 & 58.5 \\
\hline 35 & 5 & 7 & 59.5 \\
\hline \multicolumn{4}{|c|}{ Fractionated high dose } \\
\hline 50 & 25 & 2 & 60 \\
\hline 48 & 12 & 4 & 67.2 \\
\hline
\end{tabular}




\begin{tabular}{|llll|}
\hline Total dose (Gy) & No. of fractions & Dose per fraction (Gy) & BED10 \\
\hline 60 & 30 & 2 & 72 \\
\hline 50 & 10 & 5 & 75 \\
60 & 24 & 2.5 & 75 \\
65 & 25 & 2.6 & 81.9 \\
\hline
\end{tabular}

In addition, we asked all respondents which irradiation field was recommended out of the following three options: "the range where tumors existed preoperatively," "the entire orthopedic prosthesis," or "the entire affected bone."

\section{Results}

Eighty-nine radiation oncologists from 67 institutions (50\% of JROSG participating facilities) responded. The study responders' median experience in radiation oncology was 20 years (range; $2.5-40$ years). Of all the RT plans used at these institutions in 2017, 152 were eligible for this study. Except for the one RT plan with an unknown prescribed dose-fractionation, the rest 151 were enrolled in the study. There were 21 different dose-fractionation regimens ranging from $8 \mathrm{~Gy}$ in one fraction to $65 \mathrm{~Gy}$ in 25 fractions in these 151 RT plans (Supplemental table 1). Among the five classified categories of those dose-fractionation regimens, the most commonly actual used dose-fractionation was $30 \mathrm{~Gy}$ in 10 fractions $(n=75 ; 50 \%)$, followed by fractionated low dose (Fig. 1). The commonest dose-fractionation in fractionated low dose was $20 \mathrm{~Gy}$ in five fractions $(n=29 ; 19 \%)$. Single-fractioned RT was prescribed only in $3 \%$ of cases $(n=4)$, whereas the remaining $97 \%$ patients $(n=147)$ received fractioned RT.

For the four hypothetical cases, 15 different dose-fractionations were recommended by study responders, ranging from $8 \mathrm{~Gy}$ in one fraction to $60 \mathrm{~Gy}$ in 30 fractions (Supplemental table 2). For all the four cases, the most common recommended dose-fractionation was 30 Gy in 10 fractions (Fig. 2). For case 1, fractionated low dose regimens were recommended only by 17 respondents (19\%). Sixty-eight respondents (76\%) did not recommend short course RT (e.g., 8 Gy in 1 fraction or 20 Gy in 5 fractions) for Case 1. Figure 3 summarizes the reasons why these respondents regarded longer course RT more than $20 \mathrm{~Gy}$ in 5 fractions as superior to short course RT for Case 1. "Local control"was the most often cited reason $(n=37 ; 54 \%)$, followed by "incidence of re-irradiation" ( $n=23 ; 34 \%)$, and "time until first increase in pain" ( $n=13 ; 19 \%)$. Fractionated dose regimens more than 30 Gy in 10 fractions were relatively recommended in case of oligometastasis $(n=47 ; 53 \%)$, compared to those with radio-resistant tumors ( $n$ $=30 ; 34 \%)$ and those with expected long-term prognosis $(n=22 ; 25 \%)$. Fractionated intermediate dose regimens were recommended for oligometastasis (Case 2) or radio-resistant primary tumor (Case 3 ) in comparison with other cases. Especially, fractionated high dose regimens were recommended for oligometastasis $(n=11 ; 12 \%$ ), while none of the respondents recommended high dose per fraction for radio-resistant primary tumor. Fifty-nine respondents $(66 \%)$ recommended 30 Gy in 10 fractions for expected long survival (Case 4), which was similar with comparatively limited survival (Case 1) $(n=60$; 
$67 \%)$. The irradiation field recommended for PORT by the majority of responders was "the entire orthopedic prosthesis" ( $n=66 ; 74 \%)$, followed by "the range where tumors existed preoperatively" $(n=9$; $10 \%$ ) and "the entire affected bone" ( $n=8 ; 9 \%)$ (Fig. 4).

\section{Discussion}

The results of this study show that $30 \mathrm{~Gy}$ in 10 fractions has been most commonly used for PORT of metastases to the long bones in current practice in Japan. Half of the RT plans irradiated at JROSG institutes were prescribed $30 \mathrm{~Gy}$ in 10 fractions. In addition, the majority of the respondents also recommended this regimen in hypothetical cases $(67 \%$ for Case $1,45 \%$ for Case $2,53 \%$ for Case 3 , and $66 \%$ for Case 4). Our results are similar to those reported by previous retrospective studies about PORT for metastases to the long bones [8-11]. In most of these studies fractioned RT, such as 30 Gy in 10 fractions or $20 \mathrm{~Gy}$ in 5 fractions, ranging from $8 \mathrm{~Gy}$ to $56 \mathrm{~Gy}$ was used. In addition, as per the American College of Radiology (ACR) Appropriateness Criteria for Non-Spine BMs [12], which is based on multidisciplinary expert opinion, while there are no definitive data to suggest the most appropriate radiotherapy dose, $30 \mathrm{~Gy}$ in 10 fractions seems to be a reasonable option with the goal of eradicating microscopic residual disease. In the expert opinion, $8 \mathrm{~Gy}$ in 1 fraction to $35 \mathrm{~Gy}$ in 14 fractions are also equally appropriate.

Among the hypothetical cases, case 2 that described a single oligometastasis of non-small-cell lung cancer in the long bone, was mostly recommended for higher dose regimens (fractionated intermediate dose regimens: $40 \%$ or fractionated high dose regimens: $12 \%$ ) in comparison to other hypothetical cases. Recently, SAbR-COMET study [13] showed that stereotactic body radiotherapy (SBRT) for oligometastasis provided a survival benefit (standard of care alone vs. SBRT arm; 28 vs. 41 months of median overall survival). Another previous study [14] has shown that high dose prescription by SBRT yields high rates of great local control of greater than $85 \%$ for non-spine BMs. Thus, the higher dose PORT regimens might also lead to good local control as well as survival benefit in case of oligometastasis.

\section{Case 3}

in our study was identical with Case 1 except for the radio-resistant primary tumor. In current study, none of the respondents recommended high-dose-per-fraction (greater than $5 \mathrm{~Gy}$ ) for Case 3. Although RCC is traditionally reported to be radio-resistant to RT [15], it has been previously reported that high-dose-perfraction RT could overcome this relative radio-resistance in RCC [16]. Similar findings for melanoma, also considered as a radio-resistant primary tumor, have also been reported [17]. However, Rades et al reported escalation of the radiation dose beyond $30 \mathrm{~Gy}$ in 10 fractions did not significantly improve motor function and local control of metastatic spinal cord compression in case of radio-resistant tumors including renal cell carcinoma, colorectal cancer, and malignant melanoma [18]. Therefore, whether to escalate radiation dose for radio-resistant primary tumors in a palliative care setting remains unclear.

\section{Case 4}


in our study described a patient with expected long-term survival. Sixty-six percent of respondents recommended $30 \mathrm{~Gy}$ in 10 fractions for Case 4, which were similar to recommendations for Case 1 with comparatively limited survival (67\%). Fractioned intermediate dose or high dose was recommended by $24 \%$ of the respondents, which was higher than that recommended for Case 1 with limited survival (9\%). However, according to the data reported for pain relief of bone metastases for 320 patients with painful BM surviving $>52$ weeks, Van der Linden et al. reported that the responses were similar in both single and multiple fraction schedules (87\% after $8 \mathrm{~Gy}$, and $85 \%$ after $24 \mathrm{~Gy}$ ) [19]. Single fraction schedule is the standard palliative treatment for all patients with painful bone metastases, including patients with an expected favorable prognosis [19]. However, the research on optimal dose fractionation in the PORT setting should also be conducted in patients with an expected favorable prognosis.

Three-quarters of respondents recommended "the entire orthopedic prosthesis" for irradiation fields during PORT. Two retrospective studies about PORT for long bones mention about irradiation fields; Townsend et al. reported that 21 out of 25 fields (84\%) included the entire orthopedic prosthesis [8], and Drost et al. reported that 72 out of 74 fields $(97.3 \%)$ included the entire orthopedic prosthesis [10]. It is thus common in practice to include at least the entire orthopedic prosthesis.

Although the objective of the PORT for long bones is not clear, Townsend et al. and Adamietz et al. retrospectively reported the efficacy of PORT based on the functional status of the extremities $[8,11]$. Other potential objective is to reduce local progression and prevent prosthesis displacement, hence reducing the need for second surgery [10]. Our study also suggests that many Japanease radiation oncologists (54\%) considered that long-course RT was preferred in PORT for the long bones due to better "local control." However, in the palliative setting, the primary object of palliative RT is not mainly local control but benefits such as improved QOL, reduction in symptoms, and overall survival. For example, Rades et al reported on 265 patients treated with RT for metastatic spinal cord compression that 1-year local control was significantly better in long-course RT compared with short-course RT, while short-course RT and long-course RT had no significant difference with respect to the effect of RT on motor function [20].

The biggest limitation of the present study was the lack of clinical follow-up data. Especially, recommendations for hypothetical cases might not truly reflect clinical management. However, understanding the patterns of practices will be important for future clinical trials. Because there is no clear evidence for the efficacy or optimal dose-fractionation of PORT for the indication of BMs of the long bones, clinical trials to examine the efficacy or optimal dose-fractionation of PORT would be necessary in the future.

\section{Conclusion}

We found that 30 Gy in 10 fractions for the entire orthopedic prosthesis was preferred in current practice in Japan. Based primarily on local control of the tumor, most Japanease radiation oncologists regard long course RT as superior to short course RT. Fractioned high dose regimens may be preferred for the 
indication of oligometastasis, and fractioned low dose regimens may be preferred for cases with limited survival. Single fraction RT was not preferred in this palliative setting. These results will be important to future clinical trials of PORT for the indication of metastases to the long bones. Because of the lack of evidence, research should be performed to evaluate whether PORT has a beneficial palliative effect, and to identify the optimal dose-fractionation for PORT.

\section{Declarations}

\section{Ethics approval and consent to participate:}

All respondents consented to participate in this survey.

\section{Consent for publication:}

Not applicable.

\section{Availability of data and materials:}

The datasets used during the current study are available from the corresponding author on reasonable request.

Competing interests: Dr. Harada received grants from Japanese Radiation Oncology Study Group; personal fees from AstraZeneca and BrainLab, outside the submitted work. Dr. Tago received grants from Bayer Yakuhin, Ltd., Daiichi Sankyo Company, Ltd. and Eisai Co., Ltd., outside the submitted work. For the remaining authors none were declared.

\section{Funding:}

This research did not receive any specific grant from funding agencies in the public, commercial, or notfor-profit sectors.

Authors' contributions:HK and NN designed this survey and made major contributions in collecting and analyzing the data and writing the manuscript. NS was a major contributor in writing the manuscript. $\mathrm{HH}$, $\mathrm{HN}, \mathrm{JH}, \mathrm{TK}$ and TS made contributions in creating the questionnaires. AT, HW, KI, MN, MT, MF, NU, NA, SS, TT and KY collaborated in the discussion. All authors read and approved the final manuscript.

\section{Acknowledgements:}

We gratefully acknowledge the efforts of our survey respondents. We would like to thank Editage (www.editage.jp) for English language editing.

\section{References}


1. Schulman KL, Kohles J. Economic burden of metastatic bone disease. in the US Cancer. 2007;109:2334-42. https://doi.org/10.1002/cncr.22678.

2. Chow E, Nguyen J, Zhang L, Tseng LM, Hou MF, Fairchild A, et al. International field-testing of the reliability and validity of the EORTC QLQ-BM22 module to assess health-related quality of life in patients with bone metastases. Cancer. 2012;118:1457-65. https://doi.org/10.1002/cncr.26410.

3. Tubiana-Hulin M. Incidence, prevalence and distribution of bone metastases. Bone. 1991;12:9-10. https://doi.org/10.1016/8756-3282(91)90059-r.

4. Rich SE, Chow R, Raman S, Liang Zeng K, Lutz S, Lam H, et al. Update of the systematic review of palliative radiation therapy fractionation for bone metastases. Radiother Oncol. 2018;126(3):54757. https://doi.org/10.1016/j.radonc.2018.01.003.

5. Saito T, Toya R, Oya N. Pain response rates after conventional radiation therapy for bone metastases in prospective nonrandomized studies: A systematic review. Pract Radiat Oncol. 2019;9(2):81-8. https://doi.org/10.1016/j.prro.2018.11.006.

6. Talbot M, Turcotte RE, Isler M, Normandin D, lannuzzi D, Downer P. Function and health status in surgically treated bone metastases. Clin Orthop Relat Res. 2005;438:215-20. https://doi.org/10.1097/01.blo.0000170721.07088.2e.

7. Haentjens P, De Neve W, Opdecam P. Prosthesis for the treatment of metastatic bone disease of the hip: effects of radiotherapy. Bull Cancer. 1995;82:961-70.

8. Townsend PW, Smalley SR, Cozad SC, Rosenthal HG, Hassanein RES. Role of postoperative radiation therapy after stabilization of fractures caused by metastatic disease. Int J Radiat Oncol Biol Phys. 1995;31:43-9. https://doi.org/10.1016/0360-3016(94)e0310-g.

9. Wolanczyk MJ, Fakhrian K, Adamietz IA. Radiotherapy, bisphosphonates and surgical stabilization of complete or impending pathologic fractures in patients with metastatic bone disease. $\mathrm{J}$ Cancer. 2016;7(1):121-4. https://doi.org/10.7150/jca.13377.

10. Drost L, Ganesh V, Wan BA, Raman S, Chan S, Christakis M, et al. Efficacy of postoperative radiation treatment for bone metastases in the extremities. Radiother Oncol. 2017;124(1):45-8. https://doi.org/10.1016/j.radonc.2017.05.010.

11. Adamietz IA, Wolanczyk MJ. Functional recovery after surgical stabilization and postoperative radiotherapy due to metastases of long bones. Strahlenther Onkol. 2019;195(4):335-42. https://doi.org/10.1007/s00066-018-1369-0.

12. Expert Panel On Radiation Oncology-Bone Metastases. Lutz ST, Lo SS, Chang EL, Galanopoulos N, Howell DD, Kim EY, et al. ACR Appropriateness Criteria ${ }^{\circledR}$ non-spine bone metastases. J Palliat Med. 2012;15(5):521-6. https://doi.org/10.1089/jpm.2011.0512.

13. Palma DA, Olson R, Harrow S, Gaede S, Louie AV, Haasbeek C, et al. Stereotactic ablative radiotherapy versus standard of care palliative treatment in patients with oligometastatic cancers (SABR-COMET): a randomised, phase 2, open-label trial. Lancet. 2019;393(10185):2051-8. https://doi.org/10.1016/s0140-6736(18)32487-5. 
14. Erler D, Brotherston D, Sahgal A, Cheung P, Loblaw A, Chu W, et al. Local control and fracture risk following stereotactic body radiation therapy for non-spine bone metastases. Radiother Oncol. 2018;127(2):304-9. https://doi.org/10.1016/j.radonc.2018.03.030.

15. Deschavanne PJ, Fertil B. A review of human cell radiosensitivity in vitro. Int J Radiat Oncol Biol Phys. 1996;34(1):251-66. https://doi.org/10.1016/0360-3016(95)02029-2.

16. Walsh L, Stanfield JL, Cho LC, Chang CH, Forster K, Kabbani W, et al. Efficacy of ablative high-doseper-fraction radiation for implanted human renal cell cancer in a nude mouse model. Eur Urol. 2006;50(4):795-800. https://doi.org/10.1016/j.eururo.2006.03.021.

17. Konefal JB, Emami B, Pilepich MV. Malignant melanoma: analysis of dose fractionation in radiation therapy. Radiology. 1987;164(3):607-10. https://doi.org/10.1148/radiology.164.3.3112864.

18. Rades D, Freundt K, Meyners T, Bajrovic A, Basic H, Karstens JH, et al. Dose escalation for metastatic spinal cord compression in patients with relatively radioresistant tumors. Int $\mathrm{J}$ Radiat Oncol Biol Phys. 2011;80(5):1492-7. https:// doi.org/10.1016/j.jijrobp.2010.04.026.

19. van der Linden YM, Steenland E, van Houwelingen HC, Post WJ, Oei B, Marijnen CA, et al. Dutch Bone Metastasis Study Group. Patients with a favorable prognosis are equally palliated with single and multiple fraction radiotherapy: results on survival in the Dutch Bone Metastasis Study. Radiother Oncol. 2006;78(3):245-53. https://doi.org/10.1016/j.radonc.2006.02.007.

20. Rades D, Lange M, Veninga T, Stalpers LJ, Bajrovic A, Adamietz IA, et al. Final results of a prospective study comparing the local control of short-course and long-course radiotherapy for metastatic spinal cord compression. Int J Radiat Oncol Biol Phys. 2011;79(2):524-30. https://doi.org/10.1016/j.jijobp.2009.10.073.

\section{Figures}




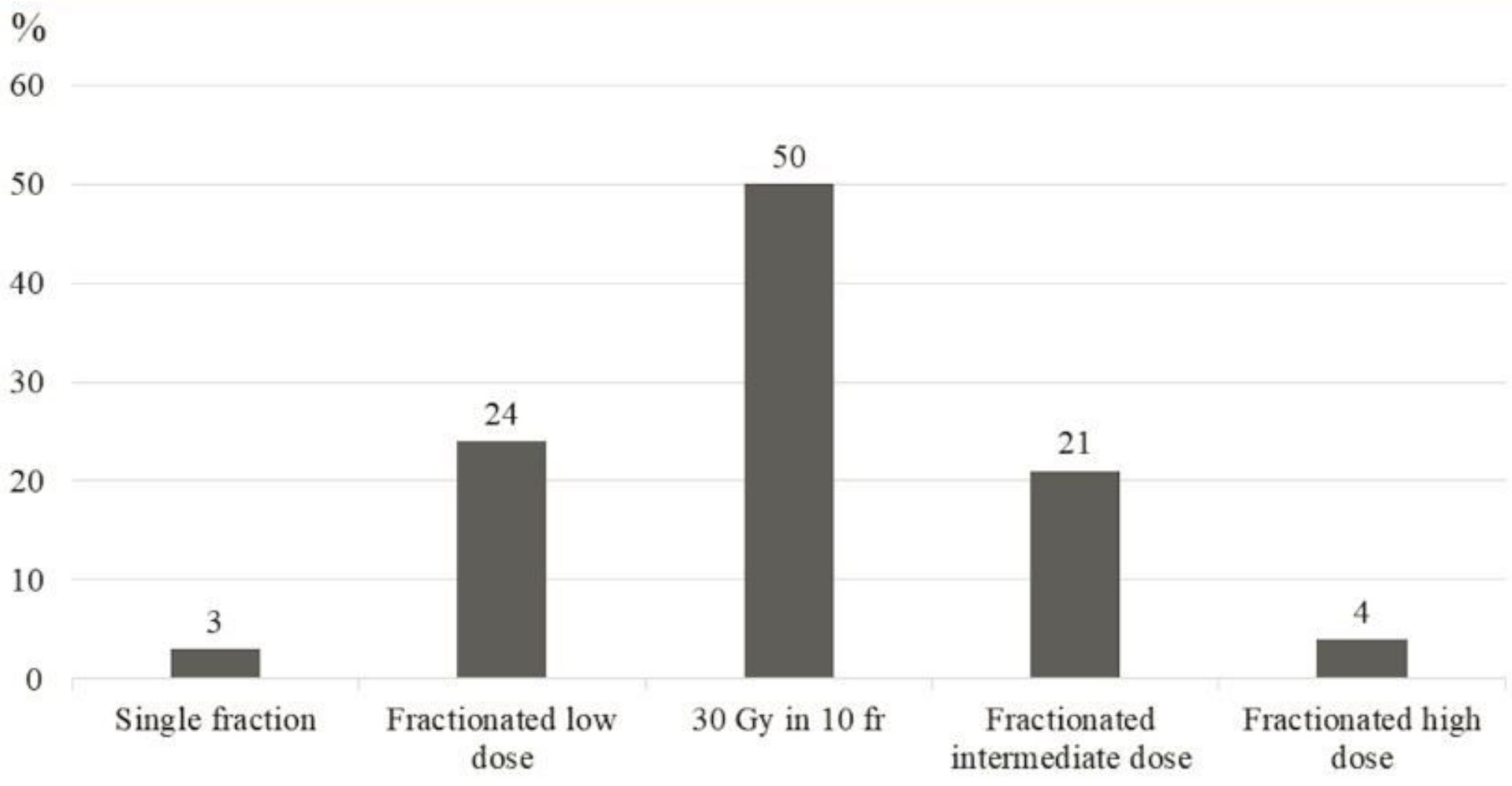

\section{Figure 1}

Dose-fractionation regimens used at the Japanese Radiation Oncology Study Group institutions Details of categories: "single fraction" includes 14.4 Gy (BED10), "fractionated low dose" includes $\geq 22.5$ Gy and $\leq 37.5 \mathrm{~Gy}$, "30 Gy in $10 \mathrm{fr}$ " includes $39 \mathrm{~Gy}$ (BED10), "fractionated intermediate dose" includes $\geq 39.2 \mathrm{~Gy}$ and $\leq 59.5 \mathrm{~Gy}$, and "fractionated high dose" includes $\geq 60 \mathrm{~Gy}$. 


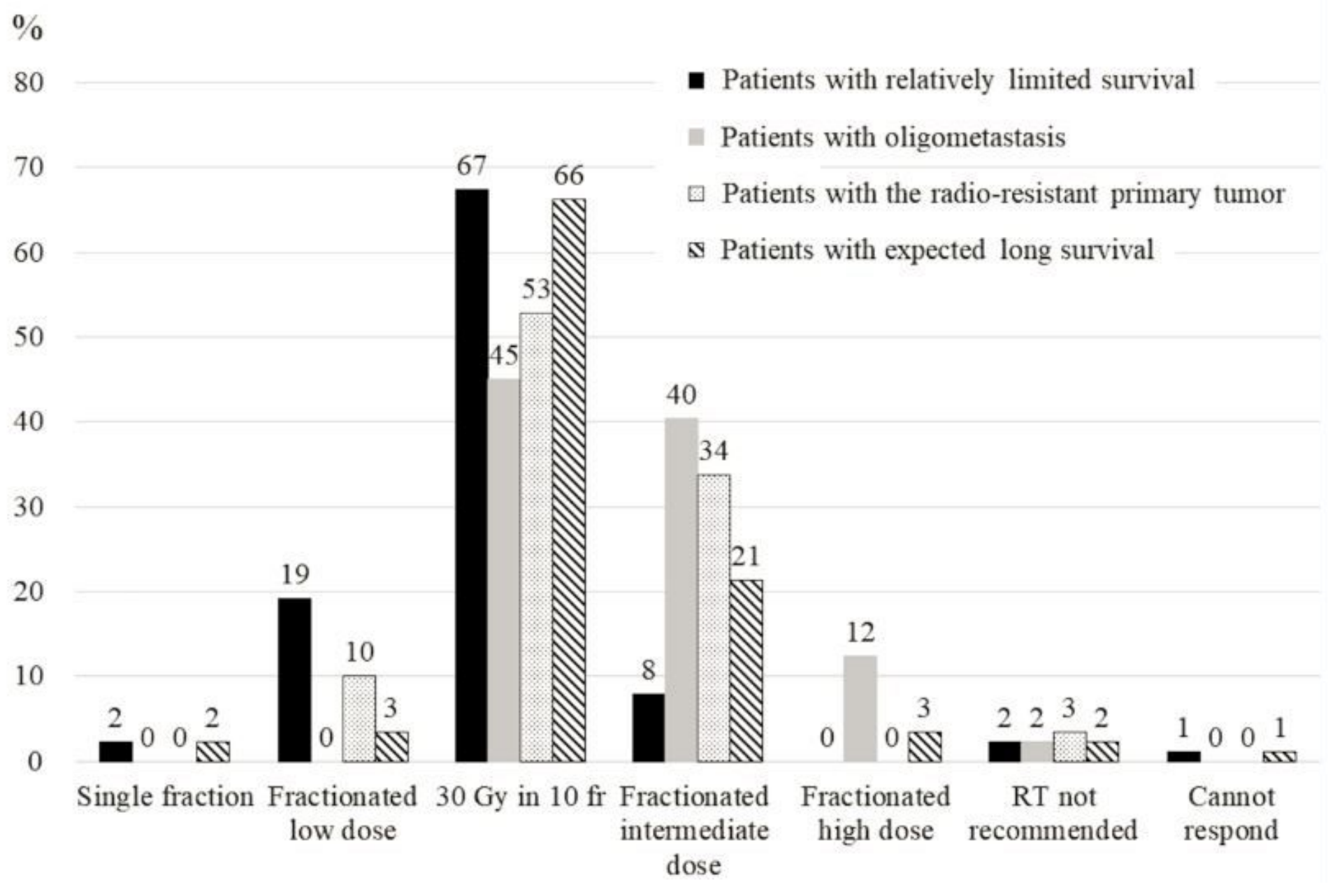

Figure 2

Dose-fractionation regimens recommended for hypothetical cases. The black bar represents the case 1 (relatively limited survival), the gray bar represents the case 2 (presence of oligometastasis), the dot pattern bar represents the case 3 (radio-resistant primary tumor), and the hatched pattern bar represents the case 4 (expected long survival). Details of categories: "single fraction" includes 14.4 Gy (BED10), "fractionated low dose" includes $\geq 22.5 \mathrm{~Gy}$ and $\leq 37.5 \mathrm{~Gy}$, "30 Gy in $10 \mathrm{fr}$ " includes $39 \mathrm{~Gy}$ (BED10), "fractionated intermediate dose" includes $\geq 39.2 \mathrm{~Gy}$ and $\leq 59.5 \mathrm{~Gy}$, and "fractionated high dose" includes $\geq 60$ Gy. 


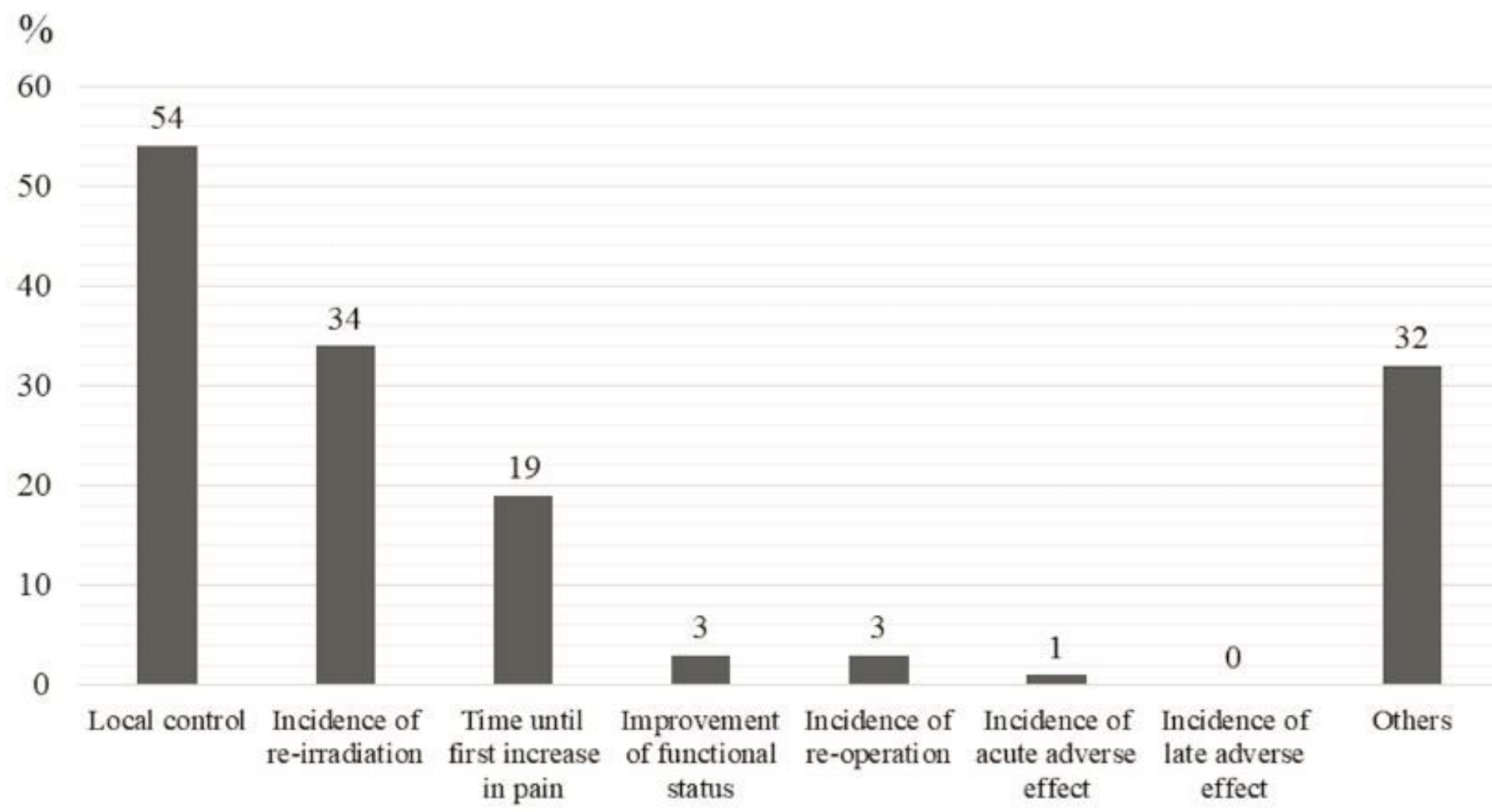

\section{Figure 3}

Respondents' reasons for not recommending short course RT for Hypothetical Case 1 Multiple choices were allowed in this question. Sixty-eight respondents recommended long course RT for Case 1.

$\%$
80
70
60
50
40
30
20
10
0

10

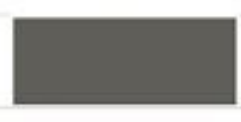

9

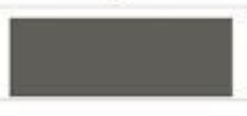

The entire orthopedic The range where tumors The entire affected bone prosthesis existed preoperatively

Figure 4 
Recommended irradiation field for PORT

\section{Supplementary Files}

This is a list of supplementary files associated with this preprint. Click to download.

- radiationoncologySupplementaltable2.docx

- radiationoncologySupplementaltable1.docx 